\title{
Recurrent Ascites: Think Outside the Box
}

\author{
Abdul I. Mahmad ${ }^{\mathrm{a}}$, Waqas Jehangir ${ }^{\mathrm{a}, \mathrm{c}}$, Jay Littlefield II ${ }^{\mathrm{b}}$, Maria J. Hoayeck ${ }^{\mathrm{a}}$, Pankaj Shirolawala ${ }^{\mathrm{a}}$, Shuvendu \\ Sen $^{\mathrm{a}}$, Abdalla Yousif ${ }^{\mathrm{a}}$
}

\begin{abstract}
Ascites is more common in cirrhotic patients. Bladder or ureteral injury is possible in surgeries involving lower abdomen or pelvic area which can mimic ascites. Intraoperative bladder puncture or ureteral nicking during abdominal surgeries is a rare but potentially serious complication. It is important to recognize these complications as early as possible to minimize damage and prevent further treatment complications. We report a case of recurrent ascites in a 65 -year-old female who underwent exploratory laparotomy for a ruptured diverticular abscess that paradoxically increased with furosemide treatment.
\end{abstract}

Keywords: Ascites; Abdominal surgeries; Paracentesis

\section{Introduction}

Ascites is more common in patients with a history of cirrhosis. Ascites is a buildup or collection of fluid in the abdominal cavity. Most often, it is due to a complication of liver cirrhosis, heart failure, and malignancy. The initial evaluation of a patient with ascites should include a history, physical evaluation, and abdominal paracentesis with ascitic fluid analysis. Appearance of the fluid, serum-to-ascites albumin gradient determination (SAAG), cell count and differential, and total protein concentration are part of the routine workup of ascites fluid. The ascitic fluid analysis helps to determine transudative versus exudative fluid collections, which can help determine the source and cause of the fluid accumulation and the presence of infection [1]. Initial treatment of ascites includes determination and treatment of the root cause of fluid accumulation. Fluid and salt avoidance is also therapeutic in decreasing the

Manuscript accepted for publication November 13, 2014

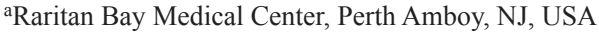

${ }^{b}$ Ross University School of Medicine, Miramar, FL, USA

${ }^{\mathrm{c} C}$ Corresponding Author: Waqas Jehangir, Department of Internal Medicine,

Raritan Bay Medical Center, Perth Amboy, NJ, USA.

Email: wjehangir@hotmail.com

doi: http://dx.doi.org/10.14740/jes247w body's water retention. Diuretics are very effectively used to therapeutically treat ascites. Another study found that a low sodium diet and diuretic therapy are initially effective in close to $90 \%$ of patients with ascites [2]. If there is an urgent need for reversal of ascites, then large volume paracentesis can be performed. Paracentesis is more rapid than diuretic therapy to remove large amounts of peritoneal fluid. Infusion of albumin was shown to help decrease mortality, when there is a removal of more than $6 \mathrm{~L}$ of fluid [3].

\section{Case Report}

A 65-year-old Caucasian female with extensive medical history inclusive of mucosa-associated lymphoid tissue nonHodgkin's lymphoma treated with radiation therapy, bronchial asthma, H. pylori gastritis, hyperlipidemia, and retinitis involving the left eye presented to emergency department with abdominal pain and weakness for 2 days. The initial complete blood count, comprehensive metabolic panel, prothrombin time, and partial thromboplastin time were normal. Liver function tests showed AST $24 \mathrm{U} / \mathrm{L}(15$ - $40 \mathrm{U} / \mathrm{L})$, ALT $8 \mathrm{U} / \mathrm{L}$ (5 - $30 \mathrm{U} / \mathrm{L})$, alkaline phosphatase $85 \mathrm{U} / \mathrm{L}(40$ - $130 \mathrm{U} / \mathrm{L})$ and total bilirubin $0.7 \mathrm{mg} / \mathrm{dL}(0.1-1.0 \mathrm{mg} / \mathrm{dL})$. CT of the abdomen showed diverticulitis with perforation with fluid, free air, and air bubbles. Percutaneous drainage was performed, and antibiotics were started. Repeat abdominal CT scan 6 days later showed worsening collection of fluid and air fluid levels in the left lower quadrant and the right upper quadrant between the loops of bowels. A laparotomy was performed with the drainage of the fluid collection from the abdominal cavity, irrigation of the peritoneal cavity, resection of the sigmoid colon and descending colon, mobilization of the splenic flexure and colostomy with a Hartmann's procedure. The procedure went uneventful and the patient recovered well.

Ten months later, the patient underwent elective reversal of the Hartmann's procedure. During surgery, the left common iliac vein bled extensively, requiring two units of packed red blood cells (PRBCs) in the operating room, and two more postoperatively. Postoperatively, the patient developed persistent ascites. CT of abdomen was then done which showed large ascites (Fig. 1). Over the next 6 weeks, percutaneous drainage was performed multiple times and eventually a pelvic drain was placed. Furosemide was prescribed and the subsequent 


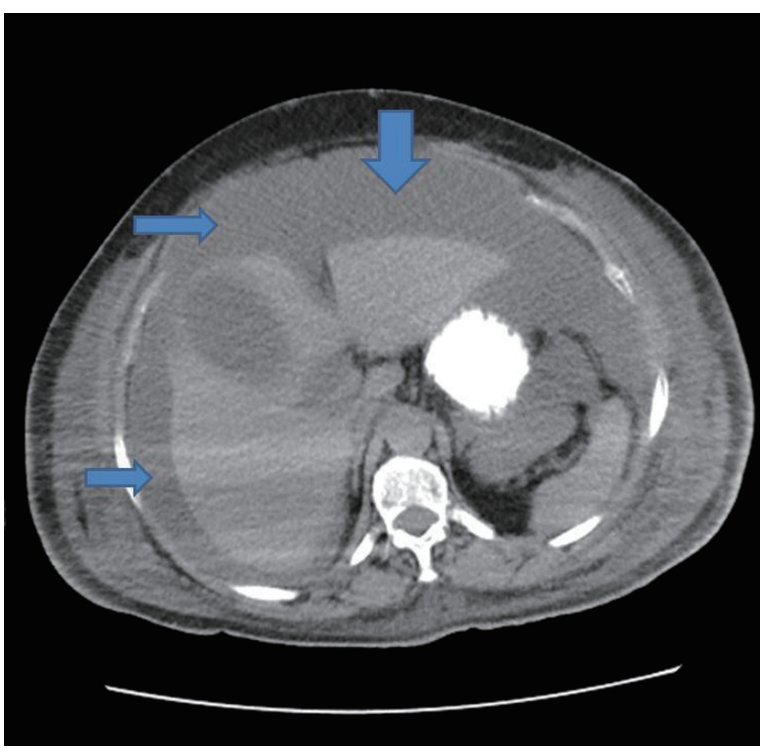

Figure 1. CT abdomen showing large ascites.

ascites worsened. Repeat $\mathrm{CT}$ was performed which showed worsening fluid in the abdomen and suggested a possible left ureter injury. The patient was then scheduled for cystoscopy and bilateral retrograde pyelogram. The procedure showed a blockage of the left ureter $5 \mathrm{~cm}$ superior to the bladder, and a patent right ureter. A percutaneous left nephrostomy tube was then placed to relieve urine drainage from the kidney. A final surgery was then performed to determine the blockage found on cystoscopy. The surgeon found the blockage in the ureter, and just superior to that was a perforation that was shown to be the source urine in the peritoneal cavity. The blockage from the ureter was then removed and the perforation was sutured closed and a stent was placed in the ureter to prevent restenosis. The patient quickly recovered and was discharged in stable condition, and had no further complaints of ascites or abdominal distension.

\section{Discussion}

Hartmann's procedure is recommended as a surgical treatment for acute conditions affecting the left-side colon which is high risk surgery and has its complications [4]. Furthermore, reversal of a left-sided end colostomy is also a high-risk major abdominal operation. Postoperative complications generally include wound infection, pneumonia, intra-abdominal abscesses, bleeding, anastomotic leak, deliriums tremens, and respiratory arrest due to overdose of narcotics, and enterocutaneous fistula [5]. Intraoperative bladder injuries do occur during surgeries more common in people going through cesarean sections but they happen rarely in the reversal of the Hartmann's procedure, which can lead to potentially serious and long-term morbidity. The complications which lead to intraoperative bladder injuries are related to urinary tract infections, prolonged surgical time or prolonged urinary catheterization. One study found the most common causes of ascites, which are mentioned in
Table 1. Causes of Ascites

\begin{tabular}{ll}
\hline Cirrhosis & $81 \%$ \\
Cancer & $10 \%$ \\
Heart failure & $3 \%$ \\
Tuberculosis & $2 \%$ \\
Dialysis & $1 \%$ \\
Pancreatic disease & $1 \%$ \\
All other causes & $2 \%$ \\
\hline
\end{tabular}

Table 1 [6].

With a large propensity of ascites being caused by cirrhosis, cancer, and heart failure (making up $94 \%$ of the cases of ascites), the workup for our patient generally was centered around the causes. But later, our patient was found to have a very rare complication of bladder perforation. Such kind of complication and injury bladder is much more likely to occur if bladder is high up. In such cases, the damage caused should not be negligent. The bladder injury is sometimes direct, sometimes caused by the mobilization of coalesced around tissues [7]. In suspected lesion, cystoscopy can help to diagnose. The correction should be made early in order to avoid complications like fistulas.

\section{Conclusion}

This case report has brought to light the other possible causes for recurrent ascites requiring multiple percutaneous paracentesis is not caused by liver disease. The report showed a case where a multidisciplinary team had to think outside of the box to determine causes of recurrent ascites, and eventually an outside of the box source was found to be the cause.

\section{Conflict of Interest}

The authors declare that there is no conflict of interests regarding the publication of this article.

\section{References}

1. Runyon BA. Management of adult patients with ascites caused by cirrhosis. Hepatology. 1998;27(1):264-272.

2. Runyon BA. Treatment of patients with cirrhosis and ascites. Semin Liver Dis. 1997;17(3):249-260.

3. Bernardi M, Caraceni P, Navickis RJ, Wilkes MM. Albumin infusion in patients undergoing large-volume paracentesis: a meta-analysis of randomized trials. Hepatology. 2012;55(4):1172-1181.

4. Roque-Castellano C, Marchena-Gomez J, Hemmersbach-Miller M, Acosta-Merida A, Rodriguez-Mendez A, Farina-Castro R, Hernandez-Romero J. Analysis of the factors related to the decision of restoring intestinal continuity after Hartmann's procedure. Int J Colorectal Dis. 2007;22(9):1091-1096. 
5. Schmelzer TM, Mostafa G, Norton HJ, Newcomb WL, Hope WW, Lincourt AE, Kercher KW, et al. Reversal of Hartmann's procedure: a high-risk operation? Surgery. 2007;142(4):598-606; discussion 606-597.

6. Runyon BA, Montano AA, Akriviadis EA, Antillon MR, Irving MA, McHutchison JG. The serum-ascites albumin gradient is superior to the exudate-transudate concept in the differential diagnosis of ascites. Ann Intern Med. 1992;117(3):215-220.

7. al-Rifaei M, el-Salmy S, al-Rifaei A, Salama A. Vesicouterine fistula--variable clinical presentation. Scand J Urol Nephrol. 1996;30(4):287-289. 